\title{
Tie-lines reveal interactions driving heteromolecular condensate formation
}

\author{
Daoyuan Qian, ${ }^{1, \dagger}$ Timothy J. Welsh, ${ }^{1,}{ }^{\dagger}$ Nadia A. Erkamp,,${ }^{1} \dagger$ Seema Qamar, ${ }^{2}$ Jonathon Nixon-Abell, ${ }^{2}$ \\ Peter St George-Hyslop, ${ }^{2,3,4}$ Thomas C. T. Michaels, ${ }^{5,1}$ and Tuomas P. J. Knowles ${ }^{1,6, *}$ \\ ${ }^{1}$ Centre for Misfolding Diseases, Yusuf Hamied Department of Chemistry, \\ University of Cambridge, Lensfield Road, Cambridge, CB2 1EW, UK \\ ${ }^{2}$ Cambridge Institute for Medical Research, Department of Clinical Neurosciences, \\ Clinical School, University of Cambridge, Cambridge CB2 OXY, UK \\ ${ }^{3}$ Department of Medicine (Division of Neurology), \\ Temerty Faculty of Medicine, University Health Network, \\ University of Toronto, Toronto M5T 0S8, Canada \\ ${ }^{4}$ Department of Neurology, Columbia University, 710 West 168th Street, New York, NY 10032, USA \\ ${ }^{5}$ Department of Physics and Astronomy, Institute for the Physics of Living Systems, University College London, London, UK \\ ${ }^{6}$ Cavendish Laboratory, Department of Physics, University of Cambridge, J J Thomson Ave, Cambridge, CB3 0HE, UK
}

\begin{abstract}
Phase separation of biomolecules can give rise to membrane-less organelles that contribute to the spatiotemporal organisation of the cell. In most cases, such biomolecular condensates contain multiple components, but the manner in which interactions between components control the stability of condensates remained challenging to elucidate. Here, we develop an approach to determine tieline gradients in ternary liquid-liquid phase separation (LLPS) systems, based on measurement of the dilute phase concentration of only one component. We studied the interaction between protein Fused in Sarcoma (FUS) and polyethylene glycol (PEG) polymer chains, and measured positive tieline gradients. This indicates that PEG drives LLPS through an associative interaction with FUS and is not an inert crowder. We further studied the interaction between PolyA RNA (3.0 $\pm 0.5 \mathrm{kDa})$ and the protein G3BP1, and using the tie-line gradient as a proxy for stoichiometry of polymers in the condensate we determined a G3BP1-to-PolyA RNA molar ratio of 1:4 in the dense phase. Our framework for measuring tie-line gradients opens up a route for the characterisation of interaction types and compositions in ternary LLPS systems.
\end{abstract}

\section{INTRODUCTION}

Biomolecular condensates play important role in cells, both in healthy physiological function and disease development $[1-5]$. These condensates form via liquid-liquid phase separation (LLPS), driven by interactions between biomolecules such as protein and RNA [6, 7]. LLPS is often studied by producing phase diagrams that show under what conditions condensates form. However, we found phase diagrams alone contain insufficient information on whether solutes co-localise in the condensate or prefer to be in separate phases, and the concept of tielines becomes important. A tie-line is defined in the following manner. Take a ternary LLPS system with two solutes - a protein and an agent - and induce phase separation by mixing a suitable amount of each. The dilute and dense phases contain certain concentrations of the protein and the agent, and we can plot the two phases as two points on the phase diagram with concentrations as their coordinates. The tie-line is the line connecting these two points. This line indicates if the solutes prefer to be in the same or different phases, which gives a positive or negative tie-line gradient respectively. Using the FloryHuggins model we found that tie-line directions directly relate to the effective interaction between solutes. In the case of a positive gradient we can further approximate

\footnotetext{
$\dagger$ These authors contributed equally

* tpjk2@cam.ac.uk
}

the gradient as the stoichiometry of solutes in the dense phase. Tie-line measurements have been performed on complex coacervates [8] by directly measuring concentrations of both solutes in dilute and dense phases, however such a measurement is challenging for protein condensates.

In this paper we demonstrate the tie-line gradient can be determined by measuring the concentration of only one solute in the dilute phase. We do not require measurement of the other solute or dense phase concentrations, both enabling investigations with solutes that cannot be easily labelled or measured and ensuring minimal artificial effect from attaching florescent tags to LLPS-inducing agents [9]. Here we studied three systems. The first and second involve the protein Fused in Sarcoma (FUS). FUS is a $75 \mathrm{kDa}$ nucleic acid binding protein known to be key for controlling gene expression across cell types $[10,11]$ and closely related to the neurodegenerative disease amyotrophic lateral sclerosis (ALS) [12]. FUS is also known to undergo LLPS in response to many stimuli including salt [13], RNA [14], and polyethylene glycol (PEG) - where PEG was previously assumed to be an inert crowder [12, 15]. We investigated LLPS of FUS with either PEG20k (20kDa) or PEG10k (10kDa). In both systems we found FUS and PEG co-localise in condensates, meaning PEG does not act as a crowder. Our third system involves the protein G3BP1, a 60kDa GTP-ase activating protein which has RNA-binding properties. G3BP1 is known the be the core protein responsible for nucleating the formation of 
stress granules $[6,16]$, a type of membrane-less organelle responsible for controlling stress response in cells [1, 17], and G3BP1 is also associated with cancer development $[18,19]$. As part of its role in stress granule formation, G3BP1 forms favourable interactions with RNA to induce formation of condensates [1]. We studied the interaction between G3BP1 and single stranded PolyA RNA $(3.0 \pm 0.5 \mathrm{kDa})$ and found that G3BP1 and the PolyA colocalize in the condensate with a molar ratio of 1:4. Our method can be widely applied to characterise interactions and compositions of condensates in other LLPS systems too.

\section{SOLUTE INTERACTION DIRECTLY RELATES TO TIE-LINE GRADIENT}

Here we use the Flory-Huggins model to investigate tie-lines. The Flory-Huggins free energy density of mixing a solvent with volume fraction $\phi_{0}$, and two different polymer solutes with lengths $N_{1}, N_{2}$ and volume fractions $\phi_{1}, \phi_{2}$ respectively, is [20-23]

$$
\begin{aligned}
\frac{f}{k_{\mathrm{B}} T}= & \phi_{0} \ln \phi_{0}+\frac{\phi_{1}}{N_{1}} \ln \phi_{1}+\frac{\phi_{2}}{N_{2}} \ln \phi_{2} \\
& +\chi_{01} \phi_{0} \phi_{1}+\chi_{02} \phi_{0} \phi_{2}+\chi_{12} \phi_{1} \phi_{2}
\end{aligned}
$$

with $k_{\mathrm{B}} T$ the unit thermal energy, $\chi_{\mu \nu}$ the effective interaction between components $\mu$ and $\nu$, and the solute volume fraction is constrained by the condition $\phi_{0}=$ $1-\phi_{1}-\phi_{2}$. The first three terms are entropic contributions that prefer mixing, while the remaining terms are interactions that can favour either mixing or phase separation, depending on values of $\chi_{\mu \nu}$. Taken together, the free energy density landscape $f\left(\phi_{1}, \phi_{2}\right)$ can be used to deduce phase-separation propensity at a particular combination of solute concentrations $\left(\phi_{1}, \phi_{2}\right)$ : if the free energy density is concave in any direction, the system is susceptible to thermal fluctuations and will spontaneously phase separate, this region of the phase diagram is the spinodal; on the other hand, a convex free energy density traps the system in a 'valley' and the mixture is locally stable. Outside the spinodal region, however, phase separation is still possible if multiple free energy minima exist and this region of metastability is denoted the binodal. A definition of tie-line gradient is natural once LLPS occurs. Denote dilute and dense phase concentrations of the two solutes as $\left(\phi_{1}^{\text {dilute }}, \phi_{2}^{\text {dilute }}\right)$ and $\left(\phi_{1}^{\text {dense }}, \phi_{2}^{\text {dense }}\right)$, the gradient of the tie-line is simply $k \equiv \frac{\phi_{2}^{\text {dense }}-\phi_{2}^{\text {dilute }}}{\phi_{1}^{\text {dense }}-\phi_{1}^{\text {dilute }}}$ since it connects both points. In the case where both solutes have low dilute phase concentrations we approximately have $k \approx \frac{\phi_{2}^{\text {dense }}}{\phi_{1}^{\text {dense }}}$, i.e. the volume ratio of solutes in the dense phase.

We use a minimal model (Appendix A1) to illustrate the phase space structure of the Flory-Huggins system. Setting solutes to unit length $N_{1}=N_{2}=1$ and assuming inert solvent $\chi_{01}=\chi_{02}=0$, the only relevant parameter is $\chi_{12} \equiv \chi$. We calculate the spinodal region analytically using the Hessian and compute the binodal and tie-lines numerically via convexification of $f\left(\phi_{1}, \phi_{2}\right)$ [24]. These two boundaries intersect and are parallel to one another at critical points. The complete phase space of the minimal model is characterised by a cooperative branch (where both solutes are in one phase) at $\chi<-8$ with positive tie-lines and a competitive branch (where solutes are in separate phases) at $\chi>2$ with negative tie-lines (figure 1). It is important to note that the phase boundaries for competitive and cooperative phase separation in the dilute regime bear great semblance to each other, so measurements of phase diagram shapes in small portions of phase space cannot determine whether cooperative or competitive interactions are drivers of LLPS.

To derive an approximate expression for the tie-line gradient $k$ we use the full Flory-Huggins expression and diagonalise the Hessian. We approximate $k$ as the gradient of the eigenvector with the smaller eigenvalue, which corresponds to the locally preferred direction of phase-separation (Appendix A2). We then show that the sign of $k, \operatorname{sgn}(k)$, is $\operatorname{sgn}(k)=-\operatorname{sgn}\left(1+2 \chi^{\Delta}\right)$ with $\chi^{\Delta} \equiv \frac{\chi_{12}-\chi_{01}-\chi_{02}}{2}$. Assuming a dilute $\phi_{1} \ll 1$ we further obtain the approximate form of $k$ as

$$
k \approx-\frac{1}{\left(1+2 \chi^{\Delta}\right) N_{1} \phi_{1}}
$$

To visualise the physical interpretation of $\chi^{\Delta}$ we recall the definition of the original $\chi_{\mu \nu}=\frac{z}{2} \frac{2 \epsilon_{\mu \nu}-\epsilon_{\mu \mu}-\epsilon_{\nu \nu}}{k_{\mathrm{B}} T}$ with $\epsilon_{\mu \nu}$ the bare contact energy between $\mu$ and $\nu$ particles and $z$ a coordination constant. Direct substitution gives $\chi^{\Delta}=\frac{\chi_{12}-\chi_{01}-\chi_{02}}{2}=\frac{z}{2} \frac{\left(\epsilon_{12}+\epsilon_{00}\right)-\left(\epsilon_{01}+\epsilon_{02}\right)}{k_{\mathrm{B}} T}$. It is evident that $\chi^{\Delta}$ signifies the energy difference between forming cooperative and competitive phases. We compare binodal tie-lines and local Hessian eigenvectors in the dilute regime of the phase diagram (figure 2) and note the two agree with each other relatively well. 


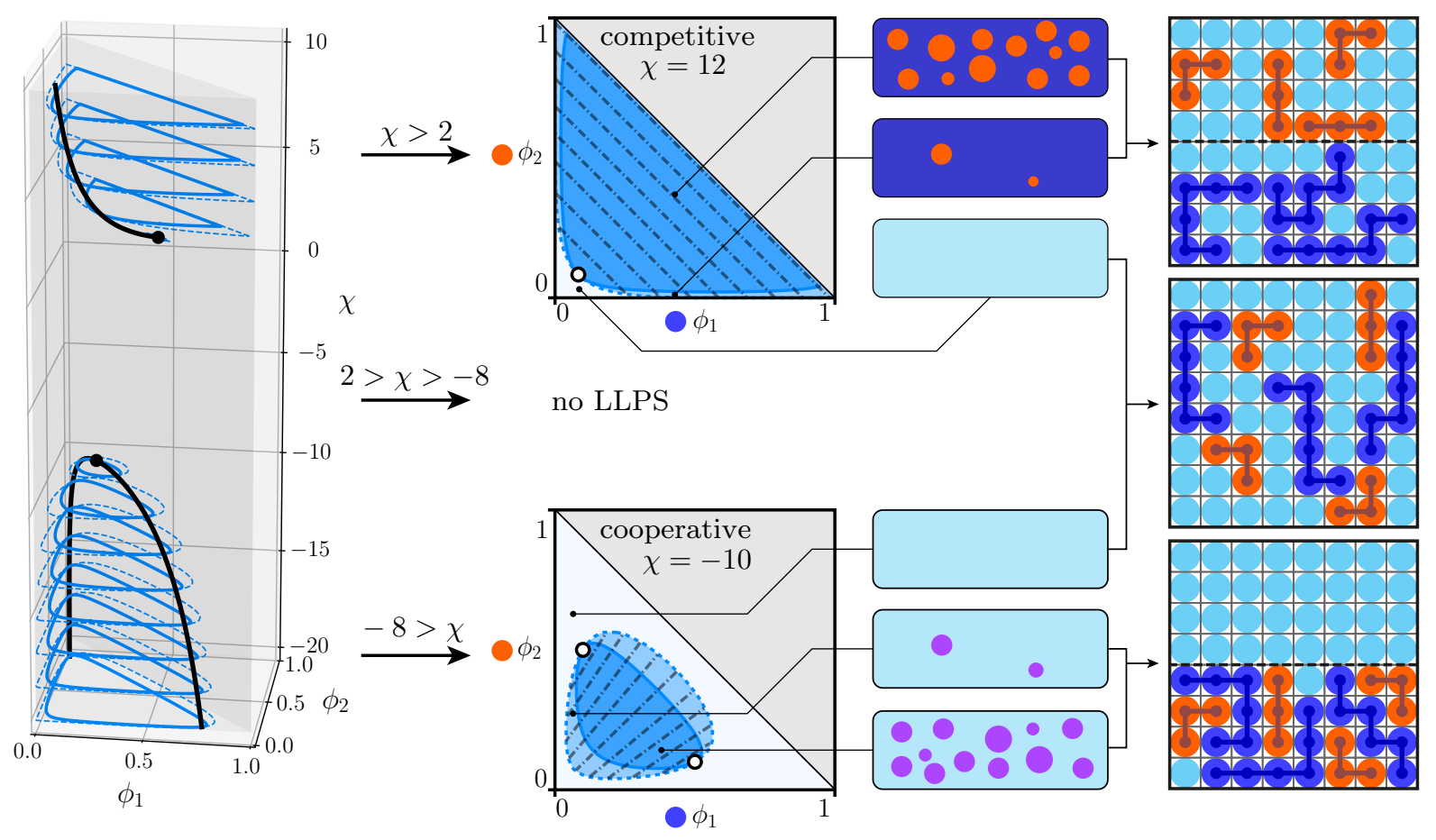

FIG. 1. Phase space structure of a ternary system. Left column: critical points (black dots and black lines), spinodal boundaries (blue solid lines) and binodal boundaries (blue dashed lines) in the $\left(\phi_{1}, \phi_{2}, \chi\right)$ space. No phase separation occurs in the $2>\chi>-8$ range. Central column: cross-sections of phase space at $\chi=12$ (top) and $\chi=-10$ (bottom), corresponding to phase diagrams with critical points (hollow circles), spinodal (dark blue region), binodal (light blue region) and tie-lines (black dashed lines) plotted. In the competitive case the individual solutes de-mix into separate compartments while in the cooperative case they co-localise in condensates. Right column: lattice model illustration of the LLPS configurations.
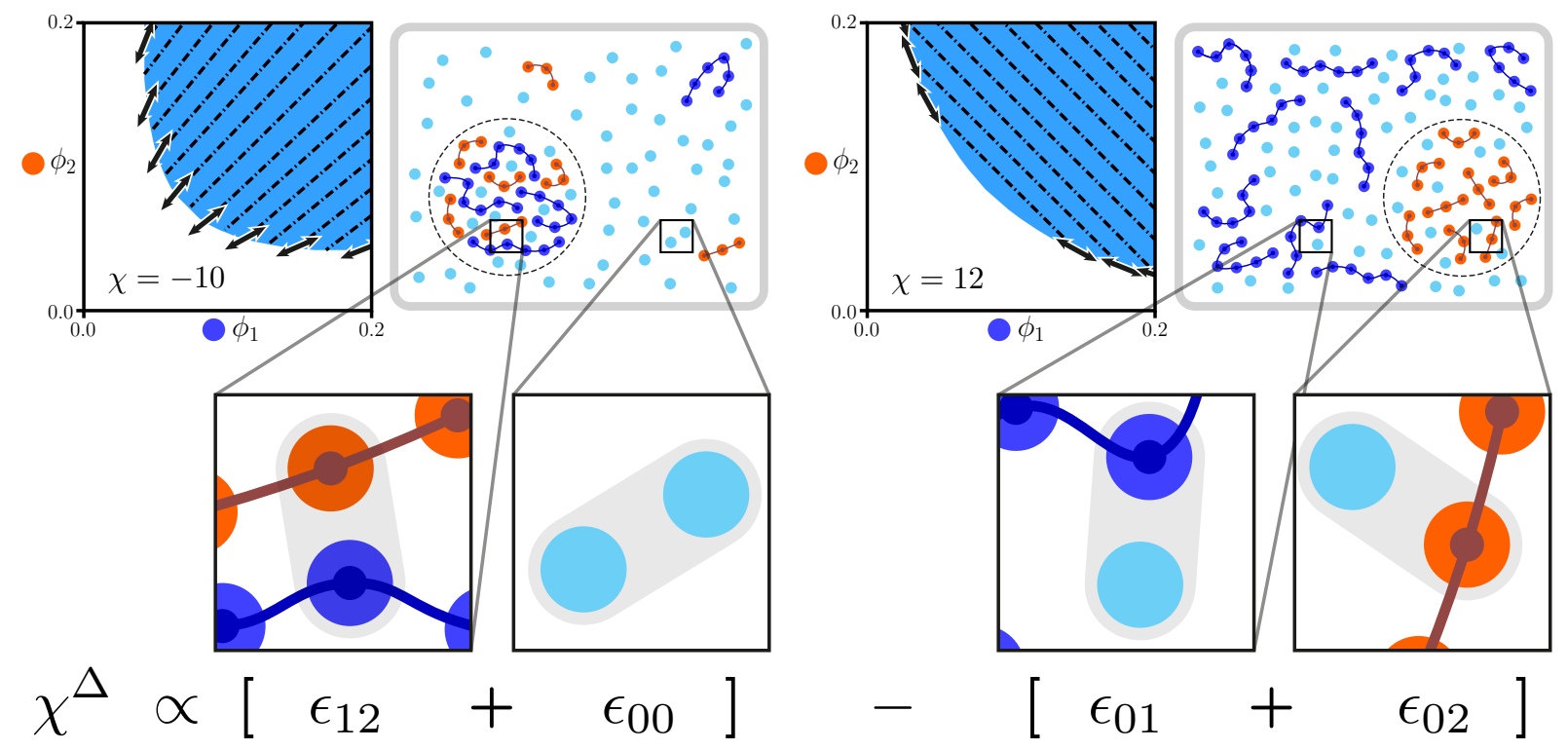

FIG. 2. Sign of the tie-line gradient is determined by $\chi^{\Delta}$. Top plots: comparison between tie-lines generated from numerical convexification (black dashed lines) and from diagonalising the Hessian (black thick arrows), with graphical illustrations of partitioning of the solutes. Dark blue regions are the binodal. Bottom zoom-ins: graphical illustration of the physical interpretation of the $\chi^{\Delta}$ parameter. $\chi^{\Delta}$ is the energy difference between forming (left) cooperative phases and (right) competitive phases. Notice homotypic interactions $\epsilon_{11}$ and $\epsilon_{22}$ do not enter $\chi^{\Delta}$, although they still affect the magnitude of the tie-line gradient. 
(A)
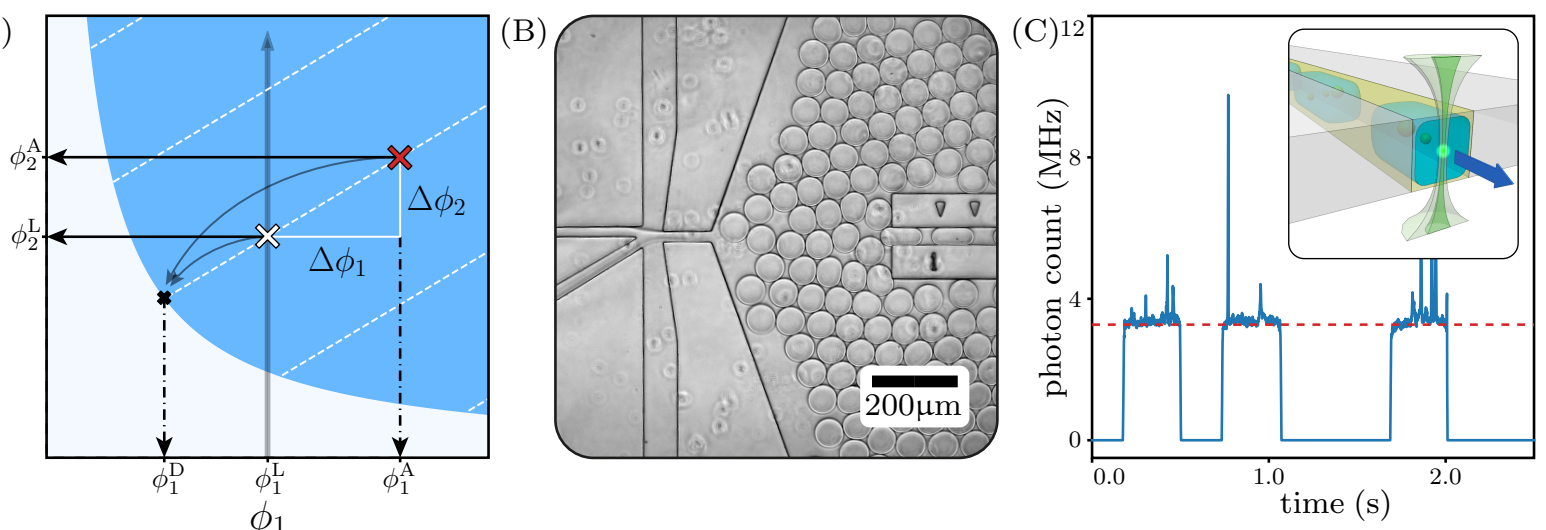
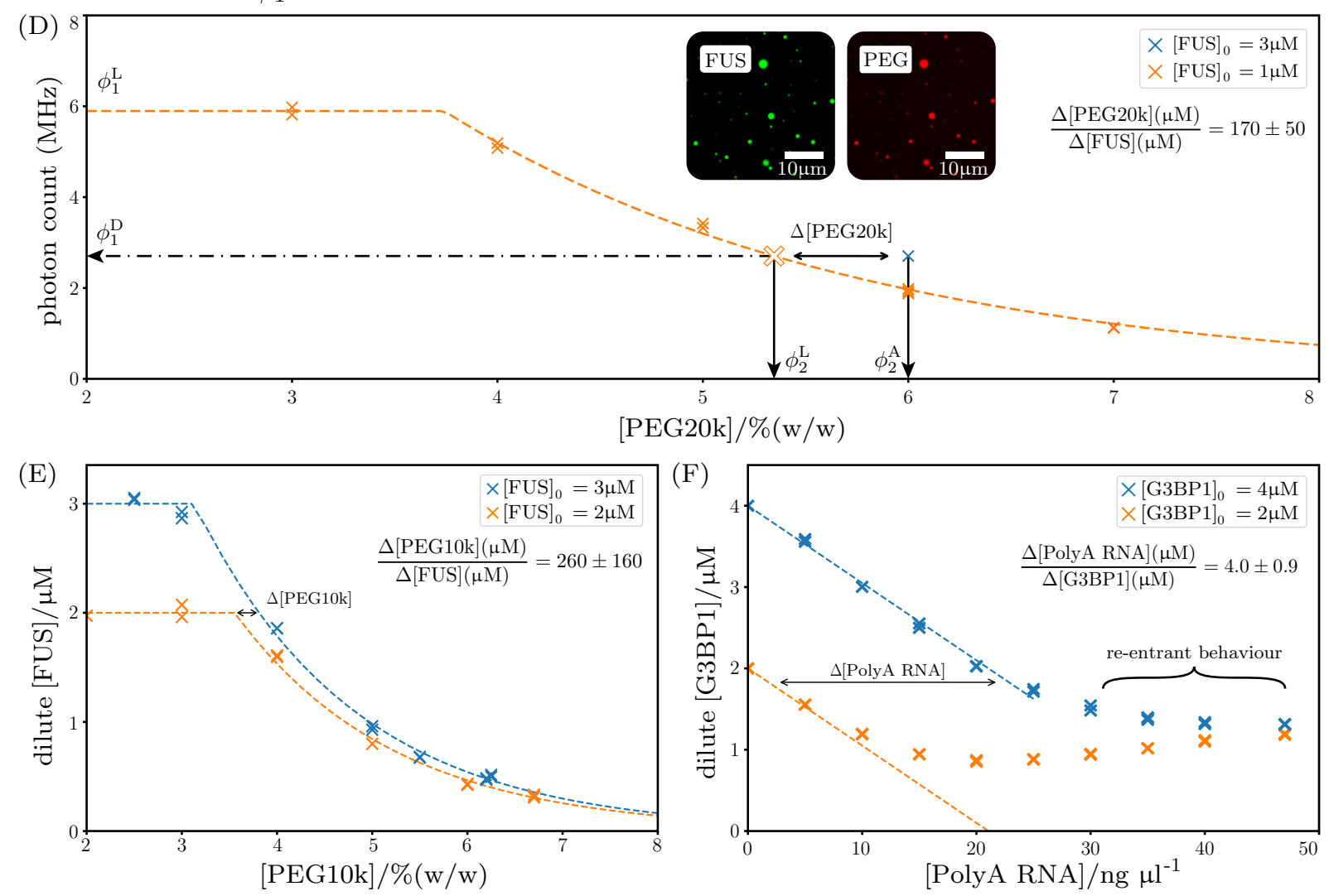

FIG. 3. (A) Illustration of anchored linescan. Blue region is the binodal with representative tie-lines (white dashed lines). The anchor is plotted as a red cross and linescan plotted as a vertical grey solid line. By comparing the dilute phase $\phi_{1}$ concentrations $\left(\phi_{1}^{\mathrm{D}}\right)$ one identifies from linescan the second point on the tie-line (white cross). The gradient can then be calculated as $k=\frac{\Delta \phi_{2}}{\Delta \phi_{1}}$. (B) Bright-field microscopy image of the microfluidic device, the droplet is generated on the left and pushed into a narrow channel on the right. Measurements were performed at the end of the channel to allow sufficient incubation time. (C) Raw signal coming from three droplets (blue solid line), characterised by plateaus from dilute phase readings and spikes from condensates passing through the confocal spot. During analysis a 3- $\sigma$ cut-off was used to filter out the spikes to give an estimate of the dilute phase photon count, averaged over $\sim 100$ droplets (red dashed line). Regions of low photon counts are gaps between droplets. Inset: 3D illustration of the channel cross-section and the confocal profile. (D) Results of anchored linescan for the FUS-PEG20k system. Solid crosses are measured values and the hollow cross is interpolated. The dashed line is a phenomenological fit. Measurement errors are comparable to marker sizes. Inset: Confocal microscopy images of condensates with both FUS and PEG20k labeled. (E) Serial linescans for the FUS-PEG10k system. Dashed lines are phenomenological fits. (F) Serial linescans for G3BP1-PolyA RNA system, showing an initial drop in dilute phase [G3BP1] due to phase separation and a rise at higher [PolyA RNA] due to the re-entrant effect. Dashed lines are linear low-[PolyA RNA] fits. Legends in (D) (F) give total starting protein concentrations in droplets. 


\section{RESULTS AND DISCUSSION}

Measurement approach. Measuring tie-lines using the dilute phase concentration of one component relies on the fact that a tie-line, by definition, is a line on which two points in the phase-separated space give the same dilute and dense phase compositions. As such, the problem reduces to finding two points in the phase diagram within the LLPS region that gives the same dilute phase concentration of one of the components - say $\phi_{1}$ assuming the phase boundary has some finite gradient. To locate two such points we can first pick a point (the 'anchor') in the phase-separated region at $\left(\phi_{1}^{\mathrm{A}}, \phi_{2}^{\mathrm{A}}\right)$ and measure the dilute phase concentration of $\phi_{1}$ as $\phi_{1}^{\mathrm{D}}$. We then pick a series of points (the 'linescan'), keeping the total $\phi_{1}$ concentration constant at some other value $\phi_{1}^{\mathrm{L}}$ while varying the $\phi_{2}$ concentration. We can then interpolate the linescan measurements and find the point with the same dilute phase concentration $\phi_{1}^{\mathrm{D}}$. This pair of points allows us to construct the tie-line by connecting them with gradient $k$ calculated as $k=\frac{\Delta \phi_{2}}{\Delta \phi_{1}}=\frac{\phi_{2}^{\mathrm{A}}-\phi_{2}^{\mathrm{L}}}{\phi_{1}^{\mathrm{A}}-\phi_{1}^{\mathrm{L}}}$ (figure 3A). Experimentally, we attached florescent tags to FUS and G3BP1 proteins and used a home-built confocal setup [25] to determine the photon count intensity from the dilute phase, which directly relate to the protein concentration (the conversion is not necessary since the $\phi_{1}^{\mathrm{D}}$ value does not enter the tie-line expression); a microfluidic device was used to trap the phase separating systems in water-in-oil droplets so that measurements could be carried out without surface effects (figure 3B, 3C and Appendix B1).

The anchored linescan is the minimal measurement needed to determine the tie-line gradient and we applied this to the FUS-PEG20k system. We then extended the method by performing multiple linescans and compared dilute phase concentrations between these, this is done in the FUS-PEG10k and G3BP1-PolyA RNA systems (Appendix B2). Proteins are produced with an insect cell line (Appendix B3) and a summary of molecular weights, densities and polymer lengths of individual components used in the following calculations are listed in Appendix B4.

Anchored linescan with FUS-PEG20k. We chose the anchor at $[\mathrm{FUS}]=3 \mu \mathrm{M}\left(\phi_{1}^{\mathrm{A}}=0.00022\right)$ and $[\mathrm{PEG} 20 \mathrm{k}]=6 \%(\mathrm{w} / \mathrm{w})\left(\phi_{2}^{\mathrm{A}}=0.053\right)$. The linescan was performed at $[\mathrm{FUS}]=1 \mu \mathrm{M}\left(\phi_{1}^{\mathrm{L}}\right)$ for $[\mathrm{PEG} 20 \mathrm{k}]$ in the range of $3.0 \sim 7.0 \%(\mathrm{w} / \mathrm{w})$. This gives $\Delta[\mathrm{FUS}]=2 \mu \mathrm{M}$. The linescan data was then fit to a phenomenological curve and we extract $\Delta[$ PEG20k] $=(0.65 \pm 0.20) \%(\mathrm{w} / \mathrm{w})(\mathrm{Ap}-$ pendix B5). Thus we obtain the tie-line gradient $k=$ $\frac{\Delta[\text { PEG 20k }]}{\Delta[\text { FUS }]}=(0.33 \pm 0.10) \frac{\%(\mathrm{w} / \mathrm{w})}{\mu \mathrm{M}}$ (figure 3D). Converting to molar ratio we get $k=170 \pm 50$. Volume and mass ratios are calculated and summarised in table $\mathrm{I}$.

The resulting positive tie-line is surprising, since we expected PEG to act as an inert crowder $[12,15]$ and it should thus have a higher concentration in the bulk to exert a positive net partial pressure on the condensates. We verified our results qualitatively by preparing condensates with fluorescently labeled FUS and PEG20k. Confocal microscopy (Appendix B6) showed that condensates are both richer in FUS and PEG20k in comparison to the dilute phase, confirming our finding that FUS and PEG20k form condensates cooperatively. Furthermore, since $\phi_{1}^{\mathrm{A}} \ll \phi_{2}^{\mathrm{A}}$ we use equation (2) to estimate $\chi^{\Delta} \approx-0.45$ so FUS and PEG20k are weakly attractive, with an effective interaction of half of $k_{\mathrm{B}} T$ between lattice sites. It should be stressed that this value is at best an order-of-magnitude estimate since we used the local Hessian to approximate the binodal tie-line, and the latter is determined globally by equilibrating both the dilute and dense phases.

Serial linescans with FUS-PEG10k. To confirm that the positive tie-line gradient is typical for $\mathrm{PEG}$ and not a coincidence due to the length of choice (20kDa), we measured the tie-line gradient for FUSPEG10k using serial linescans without anchor (figure 3E). Furthermore we also did a calibration series to map intensity to actual concentrations (appendix B1). The tie-line gradient can be extracted (Appendix $\mathrm{B} 5)$ to give $k=(0.26 \pm 0.16) \frac{\%(\mathrm{w} / \mathrm{w})}{\mu \mathrm{M}}$ and molar ratio $k=260 \pm 160$, on the same order of magnitude as the PEG20k result, indicating there is a similar cooperative interaction between FUS and PEG10k. Using equation (2) at $[\mathrm{FUS}]=1 \mu \mathrm{M}$ and $[\mathrm{PEG} 10 \mathrm{k}]=5 \%(\mathrm{w} / \mathrm{w})$ we obtain $\chi^{\Delta}=-0.74$. Moreover, we tested dextran, another carbohydrate polymer, and also found a cooperative interaction with FUS (Appendix C).

Serial linescans with G3BP1-PolyA RNA. We used single-stranded PolyA RNA $(3.0 \pm 0.5 \mathrm{kDa})$ to form protein-RNA condensates with G3BP1 and carried out serial linescans (figure $3 \mathrm{~F}$ ). The low-[PolyA RNA] region shows a steady decrease in dilute phase [G3BP1] due to phase separation, and curves up at higher [PolyA RNA] as it enters the re-entrant branch [26, 27], where the additional PolyA RNA contributes to condensate dissolution instead of formation. We performed a linear fit for the low-[PolyA RNA] branch and the tie-line gradient is $k=\frac{\Delta[\text { PolyA RNA }]}{\Delta[\text { G3BP1 }]}=(10.5 \pm 0.5) \frac{\mathrm{ng} / \mu \mathrm{l}}{\mu \mathrm{M}}$, corresponding to a molar ratio of $k=4.0 \pm 0.9$. This indicates a clear associative interaction between the G3BP1 and PolyA RNA. Using equation (2) to extract $\chi^{\Delta}$ however leads to a contact energy of the order $-70 k_{\mathrm{B}} T$ at concentrations $[\mathrm{G} 3 \mathrm{BP} 1]=4 \mu \mathrm{M}$ and $[$ PolyA RNA $]=20 \mathrm{ng} / \mu \mathrm{l}$. This is clearly unphysical and the large magnitude likely arises from underestimation of the dense phase interaction energies in the Flory-Huggins picture. G3BP1 is an RNA-binding protein and once bound, there is strong spatial correlation between the molecules and adopting a mean-field picture inevitably weakens the interactions so a much larger magnitude of $\chi^{\Delta}$ is needed to compensate for this. 
Results summary. Table I summarises the molar, volume and mass ratios of the components in condensates. For the FUS-PEG systems the main polymeric component in condensates, by all 3 metrics, is PEG. This highlights a potential caveat in using PEG to induce LLPS in vitro: the high PEG content in condensates can affect the behaviour of proteins as compared to in vivo condensates, so functional conclusions derived from in vitro experiments do not necessarily translate into in vivo results. For the G3BP1-PolyA system, we found that on average 4 PolyA molecules are present for every G3BP1 molecule. However, because PolyA has a much lower molecular weight than G3BP1, the majority of the polymers in the condensate by mass and volume is instead G3BP1.

We further note that to give a quick assessment of the sign of the tie-line gradient only two point measurements are needed in principle. One can first prepare a phaseseparated sample and measure the dilute phase concentration of one solute, and prepare another with higher total concentration of that same solute while keeping other conditions constant. If the dilute phase concentration increases after adding the solute this indicates a positive tie-line and vice versa, while a constant dilute phase concentration simply corresponds to a flat tie-line. Similar linescans can be performed in this direction as well.

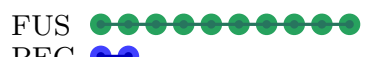

PEG ๑

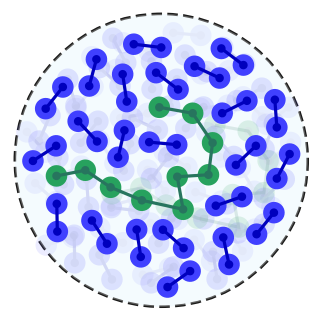

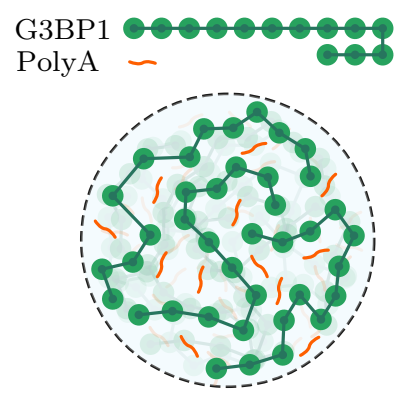

\begin{tabular}{|c|c|c|c|c|}
\hline Ratio in: & FUS & $\overline{\text { PEG20k }}$ & $\overline{\text { FUS : PEG10k }}$ & G3BP1： \\
\hline Molar & 1 & $170 \pm 50$ & $1: 260 \pm 160$ & $4.0 \pm 0.9$ \\
\hline Volume & 1 & $38 \pm 11$ & $1: \quad 29 \pm 18$ & $1: 0.12 \pm 0.03$ \\
\hline Mass & 1 & $32 \pm 9$ & $24 \pm 15$ & $1: 0.14 \pm 0.04$ \\
\hline
\end{tabular}

TABLE I. Summary of ratios of components in condensates in different units. PEG is the main polymeric component in FUS-PEG condensates in molar, volume and mass ratios while in G3BP1-PolyA RNA condensates PolyA dominate in number, and G3BP1 dominate in volume and mass.

\section{CONCLUSION AND OUTLOOK}

We have provided a theoretical framework for connecting tie-lines to molecular interactions in the study of LLPS, particularly in multicomponent biopolymer systems which are of high interest in many areas of biology. Measurement of tie-line gradients allows for a quantitative description of stoichiometry and nature of interactions between solutes in a ternary system. Since biological condensates contain multiple proteins and nucleic acid, there is a clear need for quantitative biophysical characterisation of the roles that different components play in the LLPS process and our approach provides a method for parsing the interactions apart.

As an application of the theoretical framework, we developed an experimental method using microfluidics and a home-built photon counting confocal which allowed us to monitor the dilute phase concentration of a phase separating protein, and therefore determine tie-lines by carrying out linescans across concentration series. Our findings highlighted that the protein FUS forms associative interactions with PEG with a high stoichiometry of PEG present in the condensates, and therefore contradicts previous claims that PEG is an inert crowder which lacked biophysical characterisation. Additionally, we studied the biomolecular system of G3BP1-PolyA RNA and found that our measurements agree with previous results of associative interactions between G3BP1 and RNA. We further discovered that the condensates tested had a molar ratio of $1: 4$ G3BP1:PolyA RNA. Quantitative fitting of these gradients is however challenging due to the mean-field nature of the Flory-Huggins model as well as difficulties in solving for the exact binodal. It is worth mentioning that more detailed models exist that account for strong binding between solutes such as the sticker-and-spacer model [28] and phase diagrams with tie-lines can be computed via simulation [29]. Quantitative fitting of tie-line gradients is however beyond the scope of this paper. Having developed this robust theoretical and experimental framework for quantifying biopolymeric interactions in LLPS systems, many more systems may be studied to gain further mechanistic insight to the driving forces behind LLPS. Once interactions between different components are further understood, one may be able to better design therapeutics to selectively disrupt 
or enhance LLPS of disease-relevant biomolecules with greater mechanistic insight.

Funding. This study is supported by the Harding Distinguished Postgraduate Scholar Programme (T.J.W), the Royall Scholarship (N.A.E.), Wellcome Trust Henry Wellcome fellowship 218651/Z/19/Z (J.N.A.), Canadian Institutes of Health Research (Foundation Grant and Canadian Consortium on Neurodegeneration in Aging Grant to P.St.G.H.), US Alzheimer Society Zenith Grant ZEN-18-529769 (P.St.G.H.) and Wellcome Trust Collab- orative Award 203249/Z/16/Z (P.St.G.H., T.P.J.K.).

Author Contributions. D.Q, T.J.W, N.A.E., T.C.T.M. and T.P.J.K. conceived the study. D.Q, T.J.W. and N.A.E. performed investigation. S.A., J.N.A., T.P.J.K. and P.St.G.H. provided resources. T.P.J.K. and P.St.G-H. acquired funding. D.Q., T.J.W. and N.A.E. wrote the original draft, all authors reviewed and edited the paper.

Conflict of interest. The authors report no conflict of interest.
[1] G. L. Dignon, R. B. Best, and J. Mittal, Biomolecular phase separation: From molecular driving forces to macroscopic properties, Annual Review of Physical Chemistry 71, 53 (2020).

[2] S. F. Banani, H. O. Lee, A. A. Hyman, and M. K. Rosen, Biomolecular condensates: Organizers of cellular biochemistry, Nature Reviews Molecular Cell Biology 18, 285 (2017).

[3] Y. Shin and C. P. Brangwynne, Liquid phase condensation in cell physiology and disease, Science 357, 10.1126/science.aaf4382 (2017).

[4] W. M. Babinchak and W. K. Surewicz, Liquid-Liquid Phase Separation and Its Mechanistic Role in Pathological Protein Aggregation, Journal of Molecular Biology 432, 1910 (2020).

[5] J. Söding, D. Zwicker, S. Sohrabi-Jahromi, M. Boehning, and J. Kirschbaum, Mechanisms for Active Regulation of Biomolecular Condensates, Trends in Cell Biology 30, 4 (2020).

[6] P. Yang, C. Mathieu, R. M. Kolaitis, P. Zhang, J. Messing, U. Yurtsever, Z. Yang, J. Wu, Y. Li, Q. Pan, J. Yu, E. W. Martin, T. Mittag, H. J. Kim, and J. P. Taylor, G3BP1 Is a Tunable Switch that Triggers Phase Separation to Assemble Stress Granules, Cell 181, 325 (2020).

[7] S. Boeynaems, A. S. Holehouse, V. Weinhardt, D. Kovacs, J. Van Lindt, C. Larabell, L. V. D. Bosch, R. Das, P. S. Tompa, R. V. Pappu, and A. D. Gitler, Spontaneous driving forces give rise to protein-RNA condensates with coexisting phases and complex material properties, Proceedings of the National Academy of Sciences of the United States of America 116, 7889 (2019).

[8] L. Li, S. Srivastava, M. Andreev, A. B. Marciel, J. J. De Pablo, and M. V. Tirrell, Phase Behavior and Salt Partitioning in Polyelectrolyte Complex Coacervates, Macromolecules 51, 2988 (2018).

[9] C. Uebel and C. Phillips, Phase-separated protein dynamics are affected by fluorescent tag choice., microPublication biology 2019, 139 (2019).

[10] H. Baechtold, M. Kuroda, J. Sok, D. Ron, B. S. Lopez, and A. T. Akhmedov, Human 75-kDa DNA-pairing protein is identical to the pro-oncoprotein TLS/FUS and is able to promote D-loop formation, Journal of Biological Chemistry 274, 34337 (1999).

[11] M. V. Blitterswijk and J. E. Landers, RNA processing pathways in amyotrophic lateral sclerosis, Neurogenetics 11, 275 (2010).
[12] A. Patel, H. O. Lee, L. Jawerth, S. Maharana, M. Jahnel, M. Y. Hein, S. Stoynov, J. Mahamid, S. Saha, T. M. Franzmann, A. Pozniakovski, I. Poser, N. Maghelli, L. A. Royer, M. Weigert, E. W. Myers, S. Grill, D. Drechsel, A. A. Hyman, and S. Alberti, A Liquid-to-Solid Phase Transition of the ALS Protein FUS Accelerated by Disease Mutation, Cell 162, 1066 (2015).

[13] G. Krainer, T. J. Welsh, J. A. Joseph, P. St GeorgeHyslop, A. A. Hyman, R. Collepardo-Guevara, S. Alberti, and T. P. Knowles, Reentrant Liquid Condensate Phase of Proteins is Stabilized by Hydrophobic and NonIonic interactions, Biophysical Journal 120, 28a (2021).

[14] S. Maharana, J. Wang, D. K. Papadopoulos, D. Richter, A. Pozniakovsky, I. Poser, M. Bickle, S. Rizk, J. GuillénBoixet, T. M. Franzmann, M. Jahnel, L. Marrone, Y.t. Chang, J. Sterneckert, P. Tomancak, A. A. Hyman, and S. Alberti, RNA buffers the phase separation behavior of prion-like RNA binding proteins, Science 360, 918 (2018).

[15] A. A. André and E. Spruijt, Liquid-liquid phase separation in crowded environments, International Journal of Molecular Sciences 21, 1 (2020).

[16] J. Guillén-Boixet, A. Kopach, A. S. Holehouse, S. Wittmann, M. Jahnel, R. Schlüßler, K. Kim, I. R. Trussina, J. Wang, D. Mateju, I. Poser, S. Maharana, M. Ruer-Gruß, D. Richter, X. Zhang, Y. T. Chang, J. Guck, A. Honigmann, J. Mahamid, A. A. Hyman, R. V. Pappu, S. Alberti, and T. M. Franzmann, RNAInduced Conformational Switching and Clustering of G3BP Drive Stress Granule Assembly by Condensation, Cell 181, 346 (2020).

[17] B. Wolozin and P. Ivanov, Stress granules and neurodegeneration, Nature Reviews Neuroscience 20, 649 (2019).

[18] S. Winslow, K. Leandersson, and C. Larsson, Regulation of PMP22 mRNA by G3BP1 affects cell proliferation in breast cancer cells, Molecular Cancer 12, 1 (2013).

[19] C. H. Zhang, J. X. Wang, M. L. Cai, R. Shao, H. Liu, and W. L. Zhao, The roles and mechanisms of G3BP1 in tumour promotion, Journal of Drug Targeting 27, 300 (2019).

[20] P. J. Flory, Thermodynamics of High Polymer Solutions, The Journal of Chemical Physics 10, 51 (1942).

[21] P.-G. De Gennes and P.-G. Gennes, Scaling concepts in polymer physics (Cornell university press, 1979).

[22] M. L. Huggins, Solutions of long chain compounds, The Journal of Chemical Physics 9, 440 (1941). 
[23] J. Berry, C. P. Brangwynne, and M. Haataja, Physical principles of intracellular organization via active and passive phase transitions, Reports on Progress in Physics 81, 10.1088/1361-6633/aaa61e (2018).

[24] S. Mao, D. Kuldinow, M. P. Haataja, and A. Košmrlj, Phase behavior and morphology of multicomponent liquid mixtures, Soft Matter 15, 1297 (2019), arXiv:1810.03689.

[25] G. Krainer, K. Liis Saar, W. E. Arter, and T. P. Knowles, Direct Digital Sensing of Proteins in Solution through Single-Molecule Optofluidics, Biophysical Journal 120, 114a (2021).

[26] P. R. Banerjee, A. N. Milin, M. Moosa, P. L. Onuchic, and A. A. Deniz, Reentrant phase transition drives dynamic substructure formation in ribonucleoprotein droplets Vacuolated Ribonucleoprotein Droplets RNA controls the reentrant phase transition of ribonucleoproteins (RNP) to assemble and dissolve RNP droplets. During diss, Angew Chem Int Ed Engl 56, 11354 (2017).

[27] P. Pullara, I. Alshareedah, and P. R. Banerjee, Temperature-dependent reentrant phase transition of RNA-polycation mixtures, Soft Matter , 19 (2022).

[28] A. N. Semenov and M. Rubinstein, Thermoreversible gelation in solutions of associative polymers. 1. Statics, Macromolecules 31, 1373 (1998).

[29] J.-M. Choi, F. Dar, and R. V. Pappu, LASSI: A lattice model for simulating phase transitions of multivalent proteins, PLOS Computational Biology 15, e1007028 (2019). 